\title{
3 Research Square \\ Fine Mapping and Identification of the Lobed-Leaf Gene in Radish (Raphanus Sativus L.)
}

\section{xuewei li}

Huazhong Agricultural University: National Key Laboratory of Crop Genetic Improvement https://orcid.org/0000-0003-3197-7634

\section{Chaocheng Guo}

Huazhong Agricultural University: National Key Laboratory of Crop Genetic Improvement

\section{Yao Song}

Huazhong Agricultural University: National Key Laboratory of Crop Genetic Improvement

\section{Xiaokang Zhang}

Hubei laifuzi Technology Co., Ltd

\section{Qiufang Xiong}

Wuhan Academy of Agricultural Science: The Institute of Vegetables

Kede Liu ( $\square$ kdliu@mail.hzau.edu.cn )

Huazhong Agricultural University: National Key Laboratory of Crop Genetic Improvement

\section{Research Article}

Keywords: R. sativus, the lobed-leaf trait, BSA

Posted Date: June 29th, 2021

DOI: https://doi.org/10.21203/rs.3.rs-636204/v1

License: (c) (1) This work is licensed under a Creative Commons Attribution 4.0 International License.

Read Full License 


\section{Abstract}

Leaf shape is one of the important factors affecting the yield and quality of radish (Raphanus sativus L.). In this study, an $\mathrm{F}_{2}$ population obtained from the cross of the lobed-leaf cultivar $\mathrm{J} 4$ with the serrated-leaf cultivar WA was used for genetic analysis and gene mapping of the lobed-leaf trait. The lobed-leaf trait is controlled by a single dominant nuclear gene, and dominant over the serrated-leaf trait. Through bulked segregant analysis (BSA) and genotyping by sequencing (GBS), the lobed-leaf gene was initially mapped to the genomic region from $0.66 \mathrm{Mb}$ to $8.19 \mathrm{Mb}$ on chromosome R7. By using nine insertion/deletion (INDEL) markers, the gene was further narrowed down to a $1.53 \mathrm{Mb}$ region. The comparative analysis of the collinear region between Raphanus sativus and Brassica napus identified Rs390250, an HD-Zip I transcription factor as the most probable candidate gene. Phylogenetic analysis supported that Rs390250 is an RCO (REDUCED COMPLEXITY) orthologous gene, and the single nucleotide variation (C425T) of Rs390250 which caused an amino acid substitution from serine (S) to lysine (L) in conserved leucine zipper domain, may destroy its DNA-binding function and be supposed to response for the morphological variation.

\section{Introduction}

Leaf morphology is an important agronomical trait which affects sunlight penetration, air exchange, disease occurrence, and ultimately influences the crop quality and yield (Vogel 2009; Zhu et al. 2010). Leaves of eudicots can be simple or dissected as the blade are entire or divided into leaflets, and have margins that are entire (smooth), serrated, or lobed (Hay and Tsiantis 2006; Sicard et al. 2014). Among the evolutionary history, leaf shape of many species have experienced artificial selection during domestication and breeding (Hafeez-ur-Rahman 2005). Radish (Raphanus sativus L.), an annual or biennial herb of tribe Brassicaceae, is one of the most popular vegetables all over the world. The leaves of radish cluster on a shortened stem and have serrated even lobed margins varying in sinus depth and the number of lobes. Lobed-leaf trait is important for radish growth, which can beneficial to photosynthesis enhancement and disease reduction, but there is little know about how it developed in radish. Thus, it is necessary to analyse the genetic mechanism of radish lobed-leaf trait.

Previous studies shown that lobed-leaf results from the interaction of plant hormones, transcription factors and microRNAs. Auxin is essential to the leaf margin development. It is necessary to yield auxin maxima by accumulating the auxin efflux carrier PIN1 (PIN-FORMED1) at the leaf margin to form the early tooth tips (Vernoux et al. 2000; Aloni et al. 2003). CUC2 (CUP-SHAPED COTYLEDON2) promotes the transportation of PIN1 but is repressed by auxin, thus it will accumulates at the lower-auxin-concentration locations of leaf margin (Kawamura et al. 2010). The relative distribution of CUC2 and PIN1 helps to maintain the high auxin concentration, and eventually leads to the formation of serrations, the small outgrowth at leaf margin (Furutani et al. 2004; Bilsborough et al. 2011). In addition, CUC2 is regulated by the miR319-TCPs-miR164 pathway, over-express miR319 or down-regulate TCPs (TEOSINTE BRANCHED1, CYCLOIDEA and PCF) and miR164 would increase the expression of CUC2, the leaf margin accordingly be more lobed (Nikovics et al. 2006; Koyama et al. 2010; Hasson et al. 2011). Class 
I KNOTTED1-LIKE HOMEOBOX (KNOX) are required for shoot apical meristem (SAM) maintenance and dissected leaf formation (Hay and Tsiantis 2006; Shani et al. 2009). There are four KNOX genes in Arabidopsis thaliana including STM (SHOOT MERISTEMIESS), BP(BREVIPEDICELLUS),

KNAT2 (KNOTTED-like from Arabidopsis thaliana 2), and KNAT6. It has been reported that overexpression of any the four KNOX genes would lead to deep lobes in A. thaliana (piazza et al. 2010). Meanwhile, KNOX genes were regulated by many plant hormones, high concentration of auxin inhibits the expression of KNOX1 and subsequently affects the content of gibberellic acid (GA) and cytokinin (CK), which leads to lobed margins in different degrees (Scanlon et al. 2003; Jasinski et al. 2005). It is known that LATE MERISTEM IDENTITY 1 (LMI1)-like genes play an important role in leaf shape development (Saddic et al. 2006). A. thaliana has one $L M I 7$-like gene ( $L M I 1)$, while Cardamine hirsute has three as a tandem gene triplication, LMI1, RCO (LMI1-like2, REDUCED COMPLEXITY), and LMI1-like3 (Vlad et al. 2014). Previous study showed that $L M I 1$ is required for the formation of simple serrated leaves, Imi1 plants became lobed and divided at the base of the leaf (Saddic et al. 2006). $R C O$ is able to affect the complexity of leaf shape in Brassicaceae, introducing RCO into A.thaliana would convert the simple entire leaf into lobed (Vlad et al. 2014; Sicard et al. 2014). Nowadays, LM/1-like genes have been cloned in different species, and also confirmed to regulate the leaf margin development (Chang et al. 2016; Ni et al. 2017; Andres et al. 2017; Wei et al. 2017; Hu et al. 2018; He et al. 2018; Ren et al. 2019), but the molecular mechanism is still uncertain and needs further study.

In this study, we conducted an $\mathrm{F}_{2}$ population segregating in leaf shape of radish for inheritance analysis and gene mapping. A RCO homologous gene (Rs390250) was supposed to determine the lobed leaf margin. Our study can help to analyse the molecular mechanism of $R C O$-like genes and lay a foundation for molecular marker-assisted selection of leaf shape trait in radish.

\section{Materials And Methods}

\section{Plant materials}

WA is a cytoplasmic male sterile (CMS) line with serrated leaves. $\mathrm{J} 4$ is a restorer line with lobed leaves which have deep sinuses and paired lobes. $F_{1}$ hybrids were obtained by crossing WA with $\mathrm{J} 4$ at the experimental field at the Institute of Vegetables, Wuhan Academy of Agricultural Science. $F_{2}$ population was grown at the experimental farm of Huazhong Agricultural University, Ezhou, China.

Eleven more radish varieties with various leaf shape (Table 1) were provided by the Institute of Vegetables, Wuhan Academy of Agricultural Science for single nucleotide polymorphism (SNP) analysis.

\section{Trait measurement}

The lobed and serrated leaves are obviously distinguishable when plants are at 5-6 leaf stage. The leaf shape was investigated when the two parents and the $F_{2}$ plants had 5 leaves. All the $287 F_{2}$ plants were labeled with plastic tags and their leaf shape was scored. Chi square test was used to analyze the inheritance of leaf shape. 


\section{DNA extraction and sequencing library construction}

Genomic DNA of parents and $F_{2}$ plants were extracted from about $0.25 \mathrm{~g}$ fresh leaf using the cetyltrimethyl ammonium bromide (CTAB) method. For bulked segregant sequencing (BSA-seq) and initial mapping, equal amount of genomic DNA from 20 lobed- and 20 serrated-leaf plants ware pooled to build the lobed-leaf bulk (Bk1) and the serrated-leaf bulk (BK2), respectively. Two parents and two bulks were next subjected to genotyping by sequencing (GBS) as described previously (Chen et al. 2013). Briefly, 100 ng DNA was digested with restriction enzymes Sacl and Msel. The restriction fragments were ligated to Sacl and Msel adaptors with unique barcode combinations to differentiate the samples. The ligated products of all samples were pooled and were separated on $2 \%$ agarose gel. Fragments ranging from $220 \mathrm{bp}$ to $500 \mathrm{bp}$ were recovered from agarose gel and purified with the gel purification kit (Sangon, Shanghai, China). The mixed libraries were amplified in a $50 \mu \mathrm{l}$ of volume with 50-100 ng of adaptor-ligated DNA fragments as template, $1 \times \mathrm{HF}$ buffer, $3.5 \mathrm{mM} \mathrm{MgCl}, 0.4 \mathrm{mM}$ dNTPs, 1 $U$ iProof polymerase (Bio-Rad), 5 pmol of two primers (PF:

AATGATACGGCGACCACCGAGATCTACACTCTTTC CCTACACGACGCTCTTCCGATCT and PR: CAAGCAGAAGACGGCATACGAGATCGGTCTCGGCATTCCTGCTGAACCGCTCTTCCGATCT). PCR amplification was performed as follows: $98^{\circ} \mathrm{C}$ for 2 min, followed by 11 cycles at $98^{\circ} \mathrm{C}$ for $30 \mathrm{~s}, 65^{\circ} \mathrm{C}$ for $30 \mathrm{~s}$ and $72{ }^{\circ} \mathrm{C}$ for $15 \mathrm{~s}$, and a final extension at $72{ }^{\circ} \mathrm{C}$ for $5 \mathrm{~min}$. PCR products were separated on $2 \%$ agarose gel and fragments in the size range of 270-550 bp were purified with the Sangon gel purification kit (Sangon, Shanghai, China) and sequenced on HiSeq 4000.

\section{Data analysis of BSA-seq}

To obtain the genome-wide variations between parents and between the two bulks, the sequencing reads were aligned to the reference genome of $R$. sativus (Jeong et al. 2016; http://radish-genome.org/) with BWA software. Genome-wide variations between the lobed- and the serrated-leaf bulks were obtained with GATK (McKenna et al. 2010). For each identified SNP, QTLseqr (Mansfeld and Grumet 2018) was used to calculate the SNP-index values at each quality-controlled locus. The raw sequence date were checked with the FastQC (https://www.bioinformatics.babraham.ac.uk/projects/fastqc/), and the quality of reads was controlled with Trimmomatic (Bolger 2014). The filtration parameters of GATK was "QUAL < 30.0 ॥ $\mathrm{QD}<4.0|| \mathrm{FS}>60.0 \| \mathrm{MQ}<40.0$ ". The SNP index plots and corresponding $\Delta$ (SNP index) plot of two bulks were drawn with a moving window of $2 \mathrm{Mb}$ along the physical map of the radish reference genome.

\section{Molecular marker development and genotyping}

Based on the BSA-seq data, 16 INDEL markers were designed according to 16 insertion/deletion variations of parents in the initial candidate interval. All primers used in this study are listed in Table 2. PCR amplifications were performed in a volume of $10 \mathrm{ml}$ containing $50 \mathrm{ng}$ genomic DNA, $1^{\prime}$ Taq buffer, $2 \mathrm{mM} \mathrm{MgCl} 2,0.2 \mathrm{mM}$ dNTPs, $0.2 \mathrm{mM}$ each primer and $0.25 \mathrm{U}$ Taq DNA polymerase (Vazyme, Nanjing, China). The reaction mixture was initially denatured at $94^{\circ} \mathrm{C}$ for $5 \mathrm{~min}$, followed by 40 cycles of amplification at $94{ }^{\circ} \mathrm{C}$ for $30 \mathrm{~s}, 56^{\circ} \mathrm{C}$ for $60 \mathrm{~s}$ and $72{ }^{\circ} \mathrm{C}$ for $45 \mathrm{~s}$, and a final extension at $72{ }^{\circ} \mathrm{C}$ for 5 min. 
The PCR products were separated on $6 \%$ denaturing polyacrylamide gels and stained with silver nitrate solution.

\section{Collinearity analysis}

All the predicted proteins of $R$. sativus were aligned to the $B$. napus reference genome of Darmor-bzh by BLASTP. The alignment were used as the input file of MCScanX (Wang et al. 2012) to construct the collinear segments between the radish and rapeseed genome. The collinear segment containing the candidate interval for lobed-leaf gene on $R$. sativus chromosome R7 was selected and gene-annotated.

\section{Comparative sequencing}

Based on the reference genome sequence of $R$. sativus (Jeong et al. 2016), three pairs of primers (Rs1F/Rs-1R; Rs-2F/Rs-2R; Rs-3F/Rs-3R) were designed to amplify the gene Rs390250 including $1 \mathrm{~kb}$ regulatory sequence, the $1.8 \mathrm{~kb}$ coding region and $200 \mathrm{bp}$ downstream sequence. PCR products amplified from both WA and $\mathrm{J} 4$ were separated on agarose gel and the target fragments were recovered from the gel with the Sangon gel purification kit (Sangon, Shanghai, China) and sequenced. All sequences were assembled together using Sequencher (GeneCodes, USA) to identify variations between parents.

\section{Semi-quantitative reverse-transcriptase PCR (RT-PCR)}

Total RNA of parents were extracted from fresh leaves using the TRlpure Reagent kit (Aidlab, Wuhan, China). RNA quality was check by electrophoresis on $2 \%$ agarose gel, purity and concentration were measured with Nanodrop (Thermo Scientific, USA). 2 mg RNA was reverse transcribed into cDNA using the RevertAid First strand cDNA synthesis kit (Thermo Scientific, USA). RT-PCR was performed with the cDNA to measure the relative expression level of Rs390250 in WA and J4. Gene-specific primers were obtained from a qPCR primer database at https://biodb.swu.edu.cn/qprimerdb/ (Lu et al. 2018). Actin was used as the reference gene to control the amount of input RNA.

\section{Results}

\section{Inheritance analysis}

To analyze the inheritance of leaf shape in $R$. sativus, a cross between WA, a CMS line with serrated leaf, and $\mathrm{J} 4$, a restorer line with deeply lobed leaf was conducted (Fig. 1). All $\mathrm{F}_{1}$ plants had lobed-leaves, while in $F_{2}$ population $(W A \times J 4)$, there were 68 plants with serrated leaves and 219 plants with lobed leaves, which fit an expected Mendelian segregation ratio of 1:3 $\left(\chi^{2}=0.227, p>0.05\right)$. These results indicated that the lobed-leaf is controlled by a dominant nuclear gene and dominant over the serrated-leaf.

\section{Bulked segregant sequencing of the lobed leaf gene}

To quick map the leaf shape gene, we did BSA-seq by incorporating BSA and GBS. After sequenced on HiSeq 4000 , there were 2,500,383 reads and 3,453,168 reads obtained form J4 and WA, 2,851,995 reads 
and 4,844,581 reads obtained form Bk1 and BK2, respectively. All reads were aligned to the radish reference genome (Jeong et al. 2016) to identify single nucleotide polymorphisms (SNPs) and small INDELs between parents and between two Bulks. Totally, 4,544 high-quality polymorphic loci including 3,852 SNPs and 692 INDELs were identified. For each identified SNP, the SNP index was calculated, and a corresponding $\Delta$ (SNP index) plot of two bulks was drawn along each chromosome (Fig. 2). It was found that the $\Delta$ (SNP index) in a genomic region from $0.66 \mathrm{Mb}$ to $8.19 \mathrm{Mb}$ on chromosome $\mathrm{R} 7$ exceeded the threshold of 0.5 , indicating that the leaf-shape gene may be encompassed.

\section{Rs390250 is the lobed leaf candidate gene of radish}

For further mapping, 16 INDEL markers among the initial candidate interval on chromosome R7 were designed based on the INDELs between parents. These markers were used to amplify the genomic DNA of WA and J4, 9 (RIL_1, RIL_2, RIL_3, RIL_7, RIL_11, RIL_12, RIL_14, RIL_15 and RIL_16) out of the 16 markers detected distinguishable polymorphisms (Fig. 3a). Then, these polymorphic INDEL markers were used for recombinant screening of $287 \mathrm{~F}_{2}$ individuals. Finally, the genomic region containing lobed-leaf gene was narrowed down to a $1.53 \mathrm{Mb}$ region between RIL_3 (0.87 Mb) and RIL_11 (2.40 Mb) on chromosome R7 (Fig. 3).

Radish belongs to the tribe Brassicaceae and closely related to B. rapa and B. napus, two important commercial crops as vegetables and vegetable oil. Comparative mapping using molecular markers and whole-genome alignment revealed extensive syntenic blocks all over the genomes of $R$. sativus and $B$. rapa (Li et al. 2011; Jeong 2016). To identify the candidate gene, we did whole-genome collinearity analysis between $R$. sativus and $B$. napus. The region from $0.87 \mathrm{Mb}$ to $1.3 \mathrm{Mb}$ of chromosome $\mathrm{R} 7 \mathrm{~m}$ radish which within the fine mapping region showed a good collinearity with the region between 16.3-16.8 $\mathrm{Mb}$ of chromosome $\mathrm{A} 10$ on B.napus (Fig. 3b). In the collinear region of B. napus, there were two LMI1-like genes, BnaA10g26320D (BnLL1.LMI1) and BnaA10g26330D (BnA10.LMI1) which were considered as the lobed leaf genes (Ni et al. 2017; Hu et al. 2018). Both BnLL1. LMI1 and BnA10. LMI1 are orthologous to Rs390250 (899,863-901,651 bp), one of the 53 gene models in the mapping region of radish. All these genes encode homeodomain leucine zipper class I (HD-Zip I) transcription factors. Previous results indicated that several class I HD-Zip regulators control leaf shape and size (Saddic et al. 2006). We thus listed Rs390250 as the lobed leaf candidate gene of radish.

\section{Sequence comparison analysis of Rs390250}

According to the annotation of the radish genome, Rs390250 consists of three exons and two introns, and encodes a protein with 220 amino acids. To compare the nucleotide sequences of $R s 390250$ between $\mathrm{J} 4$ and WA, a $3.0 \mathrm{~kb}$ genomic region including the $1.0 \mathrm{~kb}$ upstream regulatory sequence, the 1.8 $\mathrm{kb}$ coding region and the $0.2 \mathrm{~kb}$ downstream untranslated region of $R s 390250$ was amplified. Comparative sequencing identified three SNPs (C84G0T177A and C425T) in the coding sequence (CDS), one $149 \mathrm{bp}$ insertion in the regulatory region and one $666 \mathrm{bp}$ insertion in the first intron of Rs390250 from J4 (Fig. 4a). The 149 bp insertion in the regulatory region is a duplicate copy of a neighbouring $130 \mathrm{bp}$ 
sequence plus a $19 \mathrm{bp}$ spacer with unknown function. By searching against the transposable element (TE) database (http://pmite.hzau.edu.cn) (Chen et al. 2013), we found that the 666 bp insertion is a Mutator-like TE, which has a 9 bp target site duplication (GTTTTTAAA) and 46 bp terminal inverted repeate (GAGTTATTCTTGGGTTCACCCCCTAGGGTGAACCTTTAGGTTCACC). To detect whether the TE is co-segregate with leaf shape variations, we designed a pair of primers (Rs-3F/Rs-2R) to specifically amplify the $666 \mathrm{bp}$ insertion in the 68 serrated-leaf plants selected from the $F_{2}$ population, and found that all the 68 serrated-leaf plants did not have the 666 bp insertion while the lobed-leaf plants had (Fig. 4c), suggesting that the TE insertion in the first intron of Rs390250 co-segregated with the lobed-leaf. To reveal if the 149 bp duplication in the regulatory region affect the expression level of Rs390250, we performed RT-PCR to measure the relative expression level of Rs390250 in J4 and WA, and found that there is no significant difference between parents (Fig. 4d), indicating that the insertion is unrelated to the difference of leaf shape between $\mathrm{J} 4$ and WA .

Rs390250 encodes an HD-Zip / transcription factor. HD-Zip proteins contain a homeodomain (HD) domain and a leucine zipper (LZ) domain. The HD domain binds to double helix of target DNA, and the LZ domain helps the HD to recognize the downstream genes by forming a homo- or heterodimer structure (Tron et al. 2004). Both the HD and the LZ domains are essential for the HD-Zip I proteins to regulate the transcription of target genes. Of the three SNPs in the CDS of Rs390250, T177A is a synonymous mutation while C84G and C425T are non-synonymous mutations. The C425T mutation caused an amino acid substitution from serine (S) to leucine (L), which exactly located in the LZ domain (Fig. 4b). we aligned few of amino acid sequences of the LZ domain in LMI1-like genes from different species with various lobed-leaves, the result showed that the serine (S) residue is absolutely conserved in these species, except for the serrated-leaf WA which has a leucine (L) residue (Fig. $5 a$ ). Besides, Phylogenetic analysis of the LZ domain indicated that Rs 390250 belongs to RCO orthologous gene (Fig. 5b). Analysis of conserved domains using the NCBI Conserved Domains tool showed that both RCO and Rs390250-J4 have the HD domain and the LZ domain, while Rs390250-WA only has the HD domain but does not have the LZ domain, suggesting that the substitution of the conserved serine by leucine may destroys the structure of DNA-binding LZ domain in WA. We thus supposed that the C425T may cause the different morphological variation between J4 and WA. To verify our speculation, we amplified and analyzed the $R s 390250$ sequence from 11 more radish varieties with various leaf shape. All the serrated-leaf varieties are T/T homozygous as WA, while the lobed-leaf varieties with deep lobes are $\mathrm{C} / \mathrm{C}$ homozygous as $\mathrm{J} 4$ or heterozygous $(\mathrm{C} / \mathrm{T})$ (Table 1$)$. It is known that the rco mutant in Cardamine hirsuta resulted in a lighter leaf margin dissection (Vlad et al. 2014; Sicard et al. 2014). Thus, we speculated that the C425T transition is the causal variation resulting the serrated leaf of radish.

\section{Discussion}

Lobed leaf is a very common type of leaf shape in eudicots such as lettuce, watermelon, rapeseed etc. LMI1-like genes regulate the leaf shape formation. In previous studies, LMI1 was responsible for the 
margin development of simple leaf, while $R C O$ was required for the leaf complexity which played an essential role in leaflet formation of dissected leaf (Sicard et al. 2014). Most lobed leaf genes were homologous to $L M I 1$, while in recent years, $R C O$ was considered as a candidate gene for the lobed margin development of simple leaf, but the molecular mechanism is unclear ( $\mathrm{Ni}$ et al. 2017; Hu et al. 2018). RCO was first identified in Cardamine hirsuta. It was confirmed that $R C O$ has been lost in the evolution of $A$. thaliana, and contributing to a simple leaf form (Vlad et al. 2014). RCO affected the leaflets developing of Cardamine hirsuta mainly through local growth repression of their flanks, introducing RCO into A.thaliana, the simple serrated leaf became lobed (Vlad et al. 2014). As we know, the more lobes per leaf and the deeper sinuses between the lobes, the leaf margin dissection would be more severe. Thus, it is believed that $R C O$ also plays an important role in lobes' development of simple leaf, and the lose of $R C O$ would result in less lobed. Our results coincided with it. In this study, we identified Rs390250, an HD-Zip / transcription factor, as the lobed-leaf candidate gene, which belongs to RCO-like gene (Fig. 5). Comparative sequencing of Rs390250 in different varieties showed that all the serrated-leaf varieties have the mutant genotype as Rs390250-WA (Table. 1), which indicated that when Rs390250 mutanted, the leaf margin would be less lobed. Further more, the different allelic variation (C425T) in the conserved zipper domain which caused the amino acid substitution of Rs390250 may destroy the structure of DNA-binding leucine zipper domain in serrated-leaf varieties, thus the HD-Zip I protein in WA may fail to regulate the lobed leaf margin formation.

\section{Declarations}

\section{Funding}

Not applicable

\section{Conflicts of interest/Competing interests}

All the work described in this manuscript was original and has not been published or under consideration by any other journal. And I confirm that all authors are familiar with and agree with submission of the contents of the manuscript.

\section{Availability of data and material}

All data generated or analyzed during this study are included in this published article.

\section{Code availability}

Not applicable

\section{References}

1. Aloni R, Schwalm K, Langhans M, Ullrich $\mathrm{Cl}$ (2003) Gradual shifts in sites of free auxin-production during leaf-primordium development and their role in vascular differentiation and leaf 
morphogenesis in Arabidopsis. Planta 216:841-853. https://doi.org/10.1007/s00425-002-0937-8

2. Andres RJ, Coneva V, Frank MH (2017) Modifications to a LATE MERISTEM IDENTITY1 gene are responsible for the major leaf shapes of Upland cotton (Gossypium hirsutum L.). Proceedings of the National Academy of Sciences 114: E57-E66. https://doi.org/10.1073/pnas.1613593114

3. Bilsborough GD, Runions A, Barkoulas M, Jenkins HW, Hasson A, Galinha C, Laufs P, Hay A, Prusinkiewicz P, Tsiantis M (2011) Model for the regulation of Arabidopsis thaliana leaf margin development. Proceedings of the National Academy of Sciences 108:3424-3429. https://doi.org/10.1073/pnas.1015162108

4. Bolger AM, Lohse M, Usadel B (2014) Trimmomatic: a flexible trimmer for Illumina sequence data. Bioinformatics 30:2114-2120. https://doi.org/10.1093/bioinformatics/btu170

5. Chang LJ, Fang L, Zhu YJ, Wu HT, Zhang ZY, Liu CX, Li XH, Zhang TZ (2016) Insights into interspecific hybridization events in allotetraploid cotton formation from characterization of a generegulating leaf shape. Genetics 204:799-806. https://doi.org/10.1534/genetics.116.193086

6. Chen J, Hu Q, Zhang Y, Lu C, Kuang H (2013) P-MITE: a database for plant miniature inverted-repeat transposable elements. Nucleic Acids Res 42(D1):D1176-D1181. https://doi.org/10.1093/nar/gkt1000

7. Chen X, Li XM, Zhang B, Xu JS, Wu ZK, Wang B, Li HT, Younas M, Luo HL, Wu YF, Hu JS SN and Liu KD (2013) Detection and genotyping of restriction fragment associated polymorphisms in polyploid crops with a pseudo-reference sequence: a case study in allotetraploid Brassica napus. BMC Genomics 14:346. https://doi.org/10.1186/1471-2164-14-346

8. Hafeez-ur-Rahman, Bibi A, Latif M (2005) Okra-leaf accessions of the upland cotton (Gossypium Hirsutum L.): genetic variability in agronomic and fibre traits. Journal of Applied Genetics 46:149155

9. Hasson A, Plessis A, Blein T, Adroher B, Grigg S, Tsiantis M, Boudaoud A, Laufs DP (2011) Evolution and diverse roles of the CUP-SHAPED COTYLEDON genes in Arabidopsis leaf development. Plant Cell 23:54-68. https://doi.org/10.2307/41433803

10. Hay A, Tsiantis M (2006) The genetic basis for differences in leaf form between Arabidopsis thaliana and its wild relative Cardamine hirsuta. Nat Genet 38:942-947. https://doi.org/10.1038/ng1835

11. He DF, Xiang Z, Liang CZ, Zhu T, Abid MA, Cai YP, He JL, Zhang R (2018) Genetic variation in LBL 1 contributes to depth of leaf blades lobes between cotton subspecies, Gossypium barbadense and Gossypium hirsutum. Journal of Integrative Agriculture 17(11):2394-2404.

https://doi.org/CNKI:SUN:ZGNX.0.2018-11-004

12. Hu LM, Zhang H, Yang QY, Meng QY, Han SQ, Nwafor CC, Khan MHU, FanCC, Zhou YM (2018) Promoter variations in a homeobox gene, BnA10.LMI1, determine lobed leaves in rapeseed (Brassica napus L.). Theor Appl Genet 131:2699-2708. https://doi.org/10.1007/s00122-018-3184-5

13. Jasinski S, Piazza P, Craft J, Hay A, Woolley L, Rieu I, Phillips A, Hedden P, Tsiantis M (2005) KNOX action in Arabidopsis is mediated by coordinate regulation of cytokinin and gibberellin activities. Curr Biol 15:1560-1565. https://doi.org/10.1016/j.cub.2005.07.023 
14. Jeong YM, Kim N, Ahn BO, Oh M, Chung WH, Chung H, Jeong S, Lim KB, Hwang YJ, Kim GB, Baek S, Choi SB, Hyung DJ, Lee SW, Sohn SH, Kwon SJ, Jin M, Seol YJ, Chae WB, Choi KJ et al (2016) Elucidating the triplicated ancestral genome structure of radish based on chromosome-level comparison with the Brassica genomes. Theor Appl Genet 129:1357-1372. https://doi.org/10.1007/s00122-016-2708-0

15. Kawamura E, Horiguchi G, Tsukaya H (2010) Mechanisms of leaf tooth formation in Arabidopsis. Plant J 62:429-441. https://doi.org/10.1111/j.1365-313X.2010.04156.x

16. Koyama T, Mitsuda N, Seki M, Shinozaki K, Ohme-Takagi M (2010) TCPtranscription factors regulate the activities of ASYMMETRIC LEAVES1 and miR164, as well as the auxin response, during differentiation of leaves in Arabidopsis. Plant Cell 22:3574-3588. https://doi.org/10.1105/tpc.110.075598

17. Lu K, Li T, He J, Chang W, Zhang R, Liu M, Yu M, Fan Y, Ma J, Sun W, Qu C, Liu L, Li N, Liang Y, Wang R, Qian W, Tang Z, Xu X, Lei B, Zhang K, Li J (2018) qPrimerDB: a thermodynamics-based genespecific qPCR primer database for 147 organisms. Nucleic Acids Res 46(Database issue):D1229D1236. https://doi.org/10.1093/nar/gkx725

18. Mansfeld BN, Grumet R (2018) QTLseqr: An R package for bulk segregant analysis with nextgeneration sequencing. The plant genome 11(2). https://doi.org/10.3835/plantgenome2018.01.000

19. McKenna A, Hanna M, Banks E, Sivachenko A, Cibulskis K, Kernytsky A, Garimella K, Altshuler D, Gabriel S, Daly M, DePristo MA (2010) The Genome Analysis Toolkit: a MapReduce framework for analyzing next generation DNA sequencing data. Genome research 20(9):1297-1303. https://doi.org/10.1101/gr.107524.110

20. Nikovics K, Blein T, Peaucelle A, Ishida T, Morin H, Aida M, Laufs $P$ (2006) The balance between the MIR164A and CUC2 genes controls leaf margin serration in Arabidopsis. Plant Cell 18:2929-2945. https://doi.org/10.1105/tpc.106.045617

21. Ni X, Liu H, Huang J, Zhao J (2017) LMI1-like genes involved in leaf margin development of Brassica napus. Genetica 145:269-274. https://doi.org/10.1007/s10709-017-9963-0

22. Piazza P, Bailey CD, Cartolano M, Krieger J, Cao J, Ossowski S, Schneeberger K, He F, Meaux JD, Hall N, MacLeod N, Filatov D, Hay A, Tsiantis M (2010) Arabidopsis thaliana leaf form evolved via loss of KNOX expression in leaves in association with a selective sweep. Curr Biol 20:2223-2228. https://doi.org/10.1016/j.cub.2010.11.037

23. Ren J, Liu ZY, Du JT, Fu W, Hou A, Feng H (2019) Fine-mapping of a gene for the lobed leaf, BoLl, in ornamental kale (Brassica oleracea L. var. acephala). Mol Breeding 39(3):40. https://doi.org/10.1007/s11032-019-0944-0

24. Saddic $L A$ (2006) The $L E A F Y$ target $L M I 1$ is a meristem identity regulator and acts together with LEAFY to regulate expression of CAULIFLOWER. Development 133:1673-1682. https://doi.org/10.1242/dev.02331

25. Scanlon MJ (2003) The polar auxin transport inhibitor N-1-naphthylphthalamic acid disrupts leaf initiation, KNOX protein regulation, and formation of leaf margins in maize. Plant Physiol 133:597- 
605. https://doi.org/10.1104/pp.103.026880

26. Shani E, Burko Y, Ben-Yaakov L, Berger Y, Amsellem Z, Goldshmidt A, Sharon E, Ori N (2009) Stagespecific regulation of Solanum lycopersicum leaf maturation by class 1 KNOTTED1-LIKE HOMEOBOX proteins. Plant Cell 21:3078-3092. https://doi.org/10.1105/tpc.109.068148

27. Sicard A, Thamm A, Marona C, Lee YW, Wahl V, Stinchcombe JR, Wright SI, Kappel C, Lenhard M (2014) Repeated evolutionary changes of leaf morphology caused by mutations to a homeobox gene. Curr Biol 24:1880-1886. https://doi.org/10.1016/j.cub.2014.06.061

28. Takagi H, Abe A, Yoshida K, Kosugi S, Natsume S, Mitsuoka C, Uemura A, Utsushi H, Tamiru M, Takuno S, Innan H, Cano LM, Kamoun S, Terauchi R (2013) QTL-seq: rapid mapping of quantitative trait loci in rice by whole genome resequencing of DNA from two bulked populations. Plant $\mathrm{J}$ 74:174-183. https://doi.org/10.1111/tpj.12105

29. Tron AE, Welchen E, Gonzalez DH (2004) Engineering the loop region of a homeodomain-leucine zipper protein promotes efficient binding to a monomeric DNA binding site. Biochemistry 43:1584515851. https://doi.org/10.1021/bi048254a

30. Vlad D, Kierzkowski D, Rast MI, Vuolo F, loio RD, Galinha C, Gan XC, Hajheidari M, Hay A, Smith RS, Huijser P, Bailey CD, Tsiantis M (2014) Leaf shape evolution through duplication, regulatory diversification, and loss of a homeobox gene. Science 343:780-783. https://doi.org/10.1126/science.1248384

31. Vernoux T, Kronenberger J, Grandjean O, Laufs P, Traas J (2000) PIN-FORMED 1 regulates cell fate at the periphery of the shoot apical meristem. Development 127:5157-5165. https://doi.org/10.1007/s004290000122

32. Vogel S (2009) Leaves in the lowest and highest winds: temperature, force and shape. New Phytol 183:13-26. https://doi.org/10.1111/j.1469-8137.2009.02854.x

33. Wang Y, Tang H, DeBarry JD, Tan X, Li J, Wang X, Lee TH, Jin H, Marler B, Guo H, Kissinger JC, Paterson AH (2012) MCScanX: a toolkit for detection and evolutionary analysis of gene synteny and collinearity. Nucleic Acids Res 40:e49. https://doi.org/10.1093/nar/gkr1293

34. Wei C, Chen X, Wang ZY, Liu QY, Li H, Zhang Y, Ma JX, Yang JQ, Zhang X (2017) Genetic mapping of the $L O B E D L E A F 1$ (CILL 1) gene to a 127.6-kb region in watermelon (Citrullus lanatus L.). PloS One 12:e0180741. https://doi.org/10.1371/journal.pone.0180741

35. Zhu XG, Long SP, Ort DR (2010) Improving photosynthetic efficiency for greater yield. Annu Rev Plant Biol 61:235-261. https://doi.org/10.1146/annurev-arplant-042809-112206

36. Zou ZW, Ishida M, Li F, Kakizaki T, Suzuki S, Kitashiba H, Nishio T (2013) QTL analysis using SNP markers developed by next-generation sequencing for identification of candidate genes controlling 4methylthio-3-butenyl glucosinolate contents in roots of radish, Raphanus sativus L. PLoS One 8:e53541. https://doi.org/10.1371/journal.pone.0053541

\section{Tables}


Table 1 The genotype of the $425^{\text {th }}$ nucleotide in the coding sequence of $R s 390250$ in 11 radish varieties

\begin{tabular}{|lll|}
\hline Variety & Genotype at the $425^{\text {th }}$ nucleotide & Phenotype \\
\hline 1 & $\mathrm{~T} / \mathrm{T}$ & serrated \\
2 & $\mathrm{C} / \mathrm{C}$ & lobed \\
3 & $\mathrm{~T} / \mathrm{T}$ & serrated \\
4 & $\mathrm{C} / \mathrm{T}$ & lobed \\
\hline 5 & $\mathrm{C} / \mathrm{T}$ & lobed \\
6 & $\mathrm{C} / \mathrm{T}$ & lobed \\
\hline 7 & $\mathrm{~T} / \mathrm{T}$ & serrated \\
\hline 8 & $\mathrm{C} / \mathrm{C}$ & lobed \\
\hline 9 & $\mathrm{C} / \mathrm{T}$ & lobed \\
\hline 10 & $\mathrm{~T} / \mathrm{T}$ & serrated \\
\hline 11 & $\mathrm{C} / \mathrm{C}$ & lobed \\
\hline J4 & $\mathrm{C} / \mathrm{C}$ & lobed \\
\hline WA & $\mathrm{T} / \mathrm{T}$ & serrated \\
\hline
\end{tabular}

Table 2 Primers used in this study 


\begin{tabular}{|c|c|c|c|}
\hline Marker name & Forward primer $\left(5^{\prime}-3^{\prime}\right)$ & Reverse primer $\left(5^{\prime}-3^{\prime}\right)$ & Primers for \\
\hline RIL_1 & cgcctcatacatttgtttgg & tgctaatgttttcgttaacct & \multirow[t]{9}{*}{ gene mapping } \\
\hline RIL_2 & accaattacaaaacaacggt & taaacgaccagtacccaaag & \\
\hline RIL_3 & aagtaatggagagacctcga & gagttaaacgttctgttgca & \\
\hline RIL_7 & gacataagtctgtctggaca & tctgttgatacaaagtctctct & \\
\hline RIL_11 & atcttggtacagaactctcg & atcatgtactctcactctgc & \\
\hline RIL_12 & gtagaagatgacgccgac & gaaaccctaatcgtggctaa & \\
\hline RIL_14 & tggaagtaaaagacagacga & ggattcttgaacttttgtgct & \\
\hline RIL_15 & acctctttaactgcgtttga & agaaggatcggagaagaaga & \\
\hline RIL_16 & tcactcaaacccattatgtct & cgcaaatcacctaccaaaat & \\
\hline Rs_1 & tagagagggaagccatgttgtc & aacgttgctcttcgtagaccat & \multirow[t]{3}{*}{ gene clone } \\
\hline Rs_2 & ctttctcaccgcatgctctc & gtgctctatatcccacttctcat & \\
\hline Rs_3 & ccctatcgagtttcctgtgttc & agtctgccttcgtctaacatt & \\
\hline Rsa & cgcagtttcaagacagaatcaa & caacaagtagaagcaacacaca & RT-PCR \\
\hline Actin & atcaggaaggacttgtacggtaac & gctgagggaagcaagaatggaacc & internal control \\
\hline
\end{tabular}

\section{Figures}
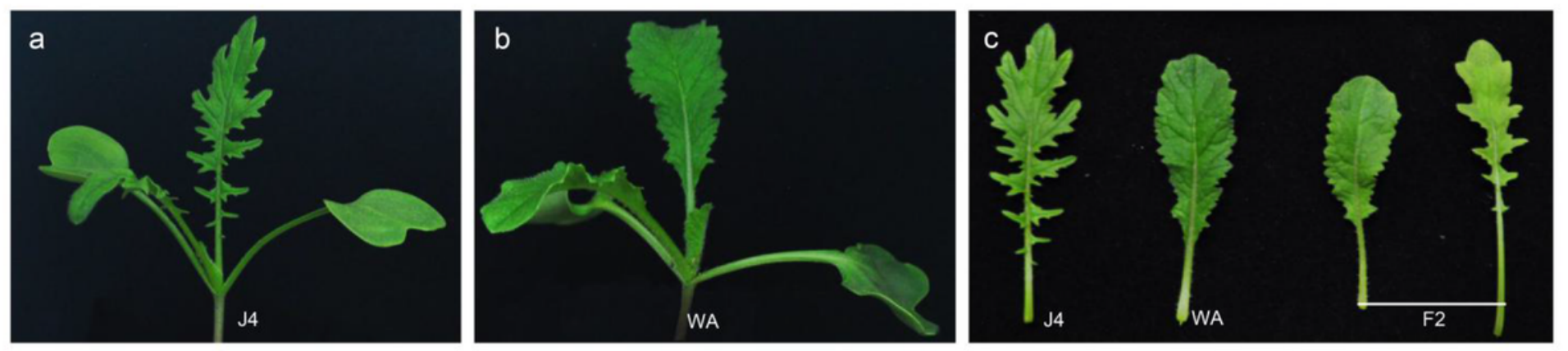

Figure 1

Morphological performance of the lobed-leaf trait at seedling stage of Raphanus sativus (a) The J4 plant at seedling stage. (b) The WA plant at seedling stage. (c) The leaves of J4, WA and some F2 plants of WA $\times \mathrm{J} 4$ at seedling stage (from left to right). 


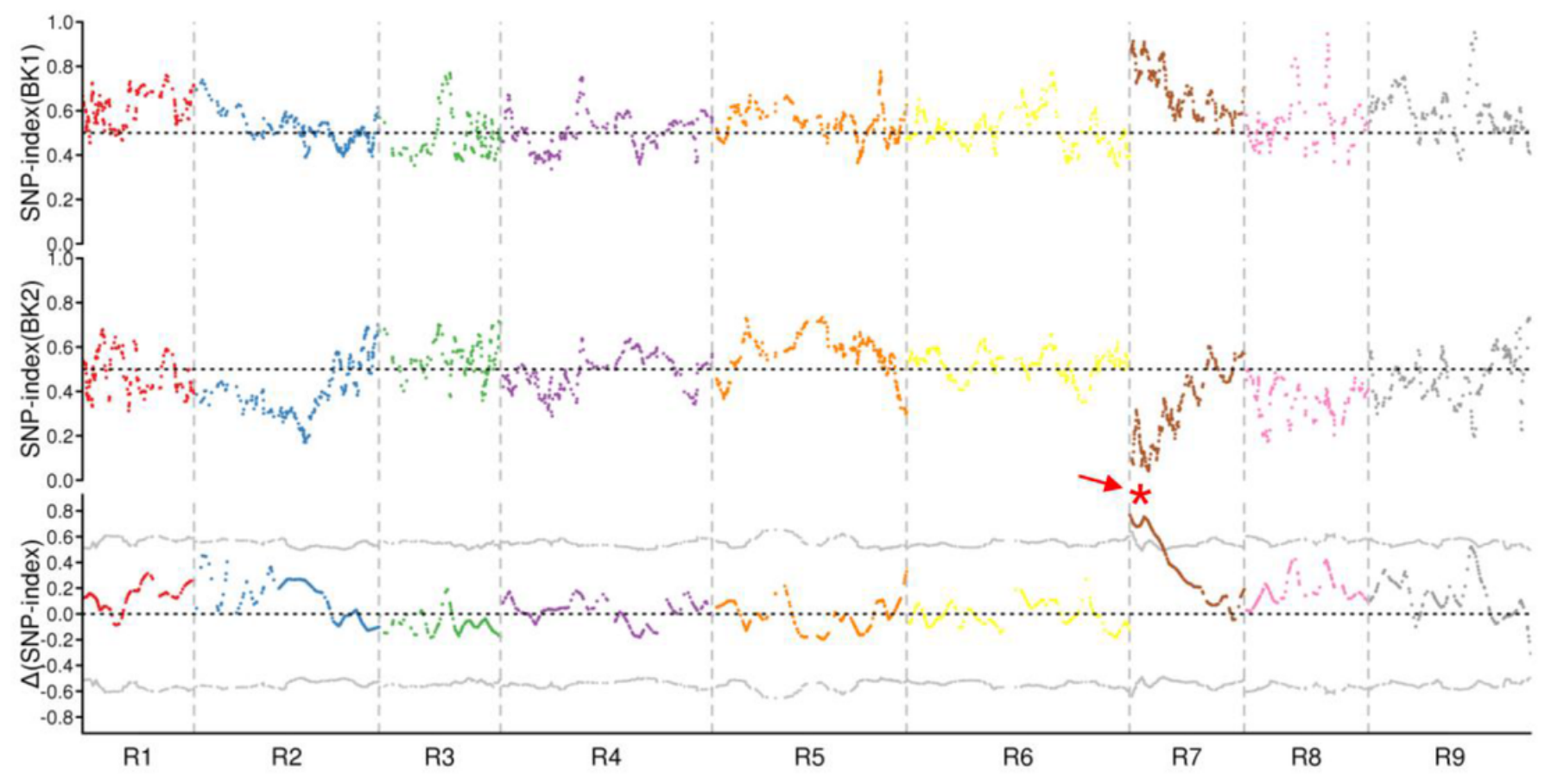

Figure 2

Genetic mapping of leaf shape gene by BSA-seq The SNP-Index attribution of the lobed-leaf DNA bulk (Bk1) and serrated-leaf DNA bulk (BK2). X-coordinate represents the location in chromosomes, Ycoordinate represents the SNP-index value and the delta SNP-index value of Bk1 and BK2 (from top to bottom). ' ' indicates the region where the delta SNP-index exceeds the threshold of 0.5. 
a

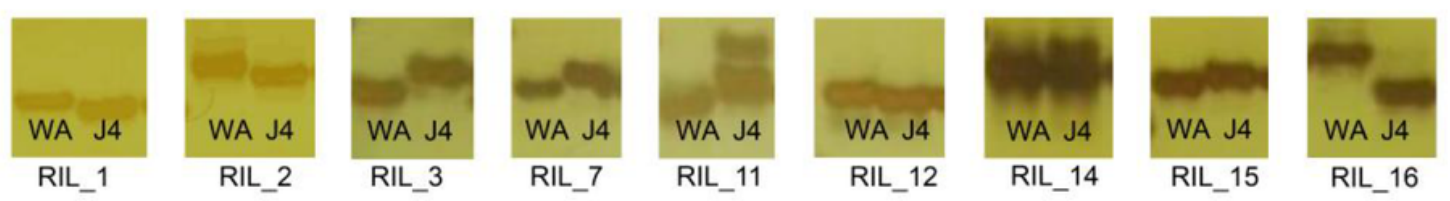

b

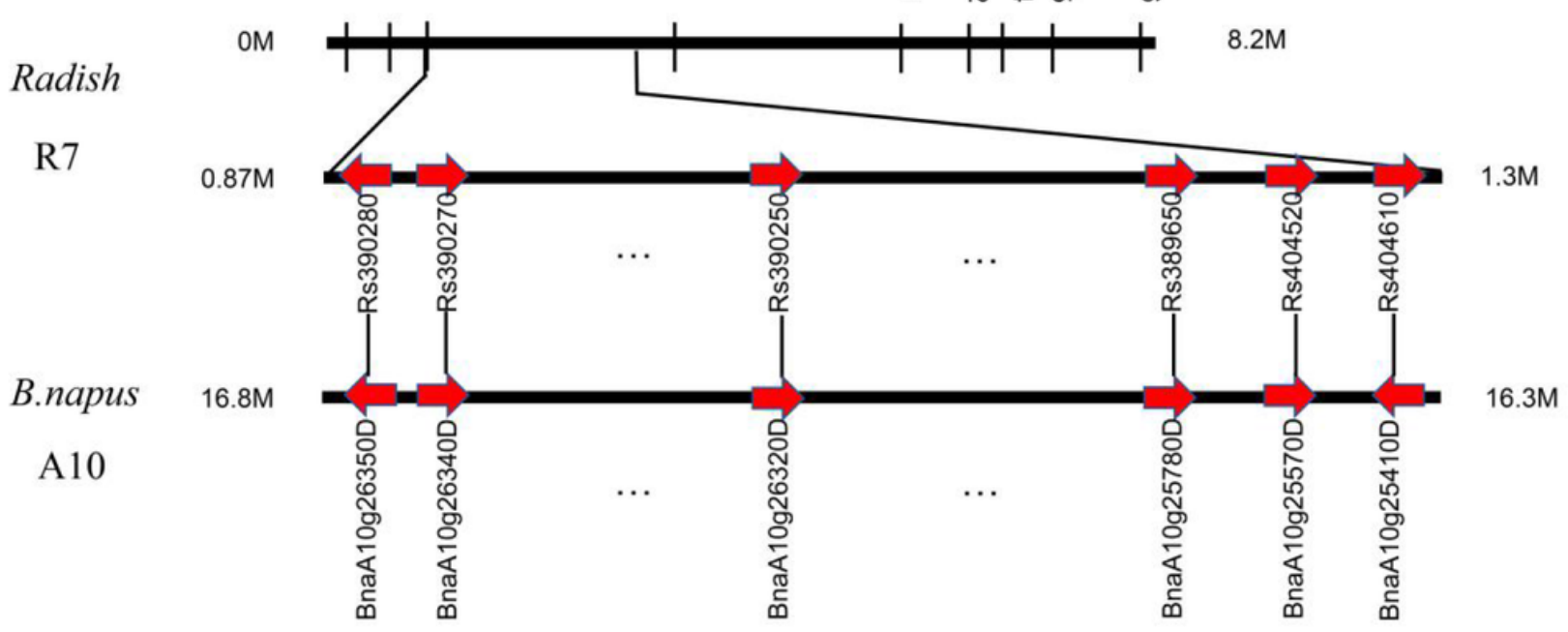

Figure 3

The lobed-leaf gene mapping in R. sativus and the collinearity analysis of candidate interval (a) Polymorphisms between WA and J4 detected by INDEL markers. (b) Collinearity between the target region of lobed-leaf gene on chromosome R7 of R. sativus and chromosome A10 of B. napus. 
Rs390250-WA

Rs390250-J4

b

Rs390250 MEWSTKSNVENMRAAFMPLQWLESNSSRSLQNFSYDPYAGNSFT PVLTQTGPVLSVPESPEKITNACQCPSIDDQMIKKKQKLTTEQLASLEQSFQEDIK Rs390250-WA MEWSTKSNVENMRAAFMPLQWLESNSSRSLQNFSYDPYAGNSFT PVLTQTGPVLSVPESPEKITNACQCPSIDDQMIKKKQKLTTEQLASLEQSFQEDIK Rs390250- J4 MEWSTKSNVENMRAAFMPLQWLESNSSSSLQNFSYDPYAGNSFTPVLTQTGPVLSVPESPEKITNACQCPSIDDQMIKKKQKLTTEQLASLEQSFQEDIK 4

Rs 390250-WA Rs $390250-\mathrm{J} 4$

Rs 390250

Rs390250-WA

Rs390250- J4

C

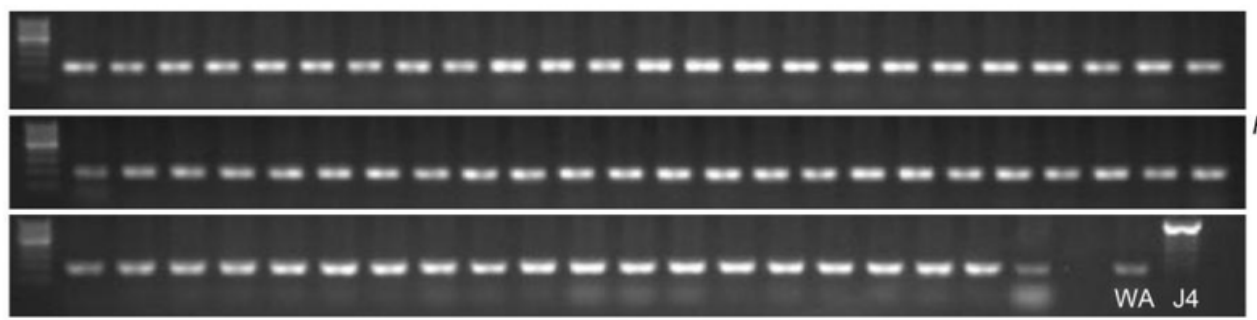

LDSDRKVKLSKELRLEPRQVAVWFQNRRARWRVKHLEESYNLIMKEYDAVSRQNQMLHDEVMKLRGIILKDHLMKRQINLNSNQMPGGSQIYGTDQYNNP LDSDRKVKLSKELRLEPRQVAVWFONRRARWRVKHLEESYNLLMKEYDAVSRQNOMLHDEVMKLRGI ILKDHLMKRQINLNSNQMPGGSQIYGTDQYNNP LDSDRKVKLSKELRLEPRQVAVWFQNRRARWRVKHLEESYNSIMKEYDAVSRQNQMLHDEVMKLRGIILKDHLMKRQINLNSNQMPGGSQIYGTDQYNNP

MCVASTCWPPLSSEQPYPW* 220 $\quad$ Exon Primers MCVASTCWPPLSSEQPYPW* 220

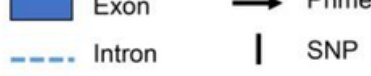

d

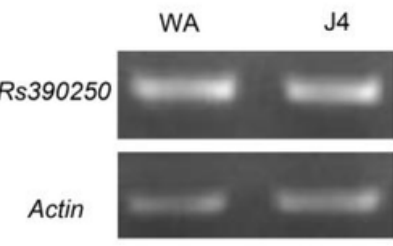

Rs-1F

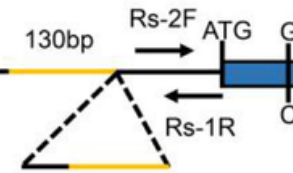

$19 \mathrm{bp} 130 \mathrm{bp}$

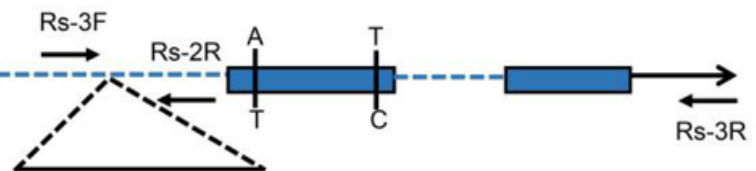

$666 \mathrm{bp}$

\section{Figure 4}

Gene structure and expression level of candidate gene Rs390250 (a) The gene structure of Rs390250; (b) Alignments of Rs390250 amino acid sequences of WA and J4. ' ${ }^{\prime}$ ' indicates amino acid change; Blue lines indicate the homeobox and red lines indicate the homeobox-associated leucine domains. (c) Primer Rs3F/Rs-2R was used to detect the co-segregation of Rs390250 with lobed-leaf trait. All 68 non-lobed leaves do not contain a 666 bp transposon sequence. (d) The expression level of Rs390250 in WA and J4. Primer Rsa is used for RT-PCR.

a

\section{Arabidopsis thaliana}

Cardamine hirsuta

Cardamine hirsuta

Cardamine hirsuta

Brassica oleracea L. var. acephala

Brassica oleracea L. var. acephala

Brassica napus

Brassica napus

Capsicum annuum

Capsella grandiflora

Capsella rubella

Raphanus sativus

Raphanus sativus
AtLMI1

ChLMI1

RCO

LMI1-like3

Bol010029

Bol010030

BnaA10g26320D

BnaA10g26330D

CaLMI1

CgRCO

CrRCO

Rs390250-J4

Rs390250-WA
KQLEQLYDSL RQEYDVVSRE KQMLHDEVCV L----KQLEQLYDSL RQEYDVVSRE KQMLHEEVKK LRAILR KHLEESYDSL RQEYDIVWRE KQMLHDEVNK LRAIIR

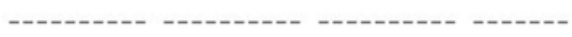

KQLEQLYDSL RQEYEVVSRE KQMLHEEVKK LRALLR KHLEESYNSL RKEYDVVSRQ NQMLHDEVMN LRGVIL KQLEQLYDSL RQEYEVVSRE KQMLHEEVKK LRALLR KQLERLYDSL KQDYDVVSRE KQKLQDEVLA LRAILK KHLEESYDSL RQEYDAVWRE KQMLHDEVKR LRAIIL KHLEESYDSL RQEYDAVWRE KQMLHDEVKR LRAIIL KHLEESYNSL MKEYDAVSRQ NQMLHDEVMK LRGIIL KHLEESYNLL MKEYDAVSRQ NQMLHDEVMK LRGIIL b

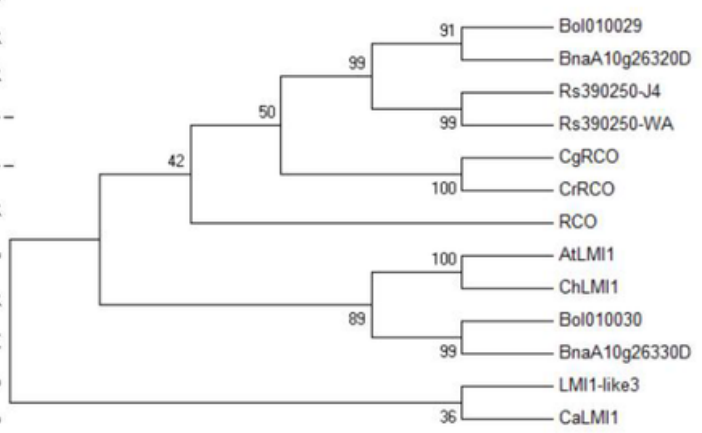

\section{Figure 5}


Comparison and phylogenetic analysis of the LMI1-like genes based on amino acid sequence (a) Alignment of amino acid sequences in the LZ domain of LMI1-like genes from different plant species with different leaf shape. The sequences from A. thaliana, B. napus and B. oleracea were download on the Brassica database (http://brassicadb.org/brad/). The others were download on the NCBI database (https://www.ncbi.nlm.nih.gov/genbank/) by searching their genbank accession numbers: ChLMI1 (KF939590), RCO (KF939591), LMI1-like3 (KF939592), CaLMI1 (PHT71668), CgRCO (KM214233) and CrRCO (KM214232). The arrow indicates the position of the absolutely conserved amino acid. (b) Phylogenetic analysis of the LMI1-like genes based on amino acid sequence from different plant species. 